\title{
MORPHOGENESIS OF MAXILLARY SINUSES IN INFANTS, DURING EARLY AND FIRST CHILDHOOD
}

DOI: 10.36740/WLek202002109

\author{
Nataliya B. Kuzniak', Larisa Ya. Fedoniuk², Antonina M. Pryshlyak², Olena I. Skyba², Oksana M. Yarema², \\ Alina I. Dovgalyuk' ${ }^{2}$, Nataliya P. Penteleichuk ${ }^{1,3}$, Vladyslav A. Smiianov ${ }^{3}$ \\ 'HSEE "BUKOVINIAN STATE MEDICAL UNIVERSITY", CHERNIVTSI, UKRAINE \\ 2. HORBACHEVSKY TERNOPIL NATIONAL MEDICAL UNIVERSITY, TERNOPIL, UKRAINE \\ ЗSUMY STATE UNIVERSITY, SUMY, UKRAINE
}

\begin{abstract}
The aim: To study the peculiarities of the structure and to see the development of maxillary sinuses in infants, during the early and first childhood periods of human ontogenesis. Materials and methods: The study of the peculiarities of the development and formation of the maxillary sinuses' wall was carried out on 50 species of the upper jaws, turtles and sections of the head of the human corpses during different periods of ontogenesis (in infants, during early and first childhood) with the help of histological examination, preparation, CT scan, radiography and morphometry.

Results and conclussions: In infants and during the early and first childhood periods of human ontogenesis in the MS there are changes in both quantitative and qualitative nature. In infants (10 days - 1 year), maxillary sinuse is located more lateral to the basis of the lower nasal concha. In this period, it begins to form its lower wall, which in the form of a narrow strip invaginates into the alveolar process. The growth of the sinus is due to the protrusion of the external wall in the direction of the zygomatic bone. The wall of the maxillary sinuse is covered with mucosa (respiratory mucous membrane), which is lined by pseudostratified columnar ciliated epithelium, which is located on the basement membrane. X-ray examination of maxillary sinuse in infants shows that it is a pear-shaped in the anterior projection. Clearly the following walls of the sinuses are visible: the upper, the lower, one, which is arched-shaped, the front and the median, which in lower part are crossed as arch to each other.

Investigation of biological specimens of the maxillofacial area of the early childhood period (1-3 years) showed that maxillary sinuses in all specimens are determined more laterally to the basis of the lower nasal concha. Its vertical dimension is 7.5-8.0 mm, transverse - 5.7-6.0 mm, anterior-posterior - 13.9-14.5 mm. X-ray examination of maxillary sinuse in early childhood in the front projection shows that it has an oval shape. There are the following walls of the sinus: upper, lower, front and middle.

The study of biological specimens of the facial area in the period of the first childhood (4-7 years) has been established that the configuration of maxillary sinuse is changing. All walls are determined, but the upper wall is rather short, and the front wall is narrow. The median wall has the most prominent development. On X-rays in the anterior projection, a slight extension of the maxillary sinuse is noted laterally.

Therefore, one should thoroughly study the peculiarities of the development and structure of the walls of the maxillary sinuses in order to prevent the development of complications and to achieve the treatment of inflammatory diseases of the MS in an optimal term.
\end{abstract}

KEY WORDS: maxillary sinuses, morphogenesis, infants, early childhood, first childhood

Wiad Lek. 2020;73(2):254-258

\section{INTRODUCTION}

The problem of purulent sinusitis in children has been and continue to be actual problem, because it is a widespread disease, it can cause rhizogenic complications and the development of pathology from other organs and systems [ 1 , 2]. Inflammation in the maxillary sinus can be a source of infectious sensitization, primarily of bronchi and lungs, it may be the causative agent of pathological reflex impulses, cause severe complications and lead to fatal cases [3,4]. In addition, up to $50 \%$ of children with upper maxillary sinusitis continue to be ill also in adulthood, sinusitis complicates up to $5 \%$ of upper respiratory infections in children, but more than $40 \%$ of them finished independently $[5,6]$.

In the mechanisms of development of the most of pathological processes, the additional individual peculiarities of the organism become important. They loaded down at the embryogenesis, the morphological substrate of them is a constitution. On the background of the current propensity to develop a specific pathology, not only the congenital features of the structure and size of the maxillary sinuses, but also the constitutional properties of the organism as a whole, achieved as a result of the implementation of an individual genetic program of development in specific environments [7].

The increase in the number of patients with acute purulent maxillary sinusitis, the complexity to diagnose the causes of its development, the severity of postsurgical complications, neurological disorders, which are associated with trauma of the second branch of the trigeminal nerve during trepanation of the sinus, disorders in the innervation of the teeth, disorders of the olfactory and nasal breathing require a detailed study of the features of the 


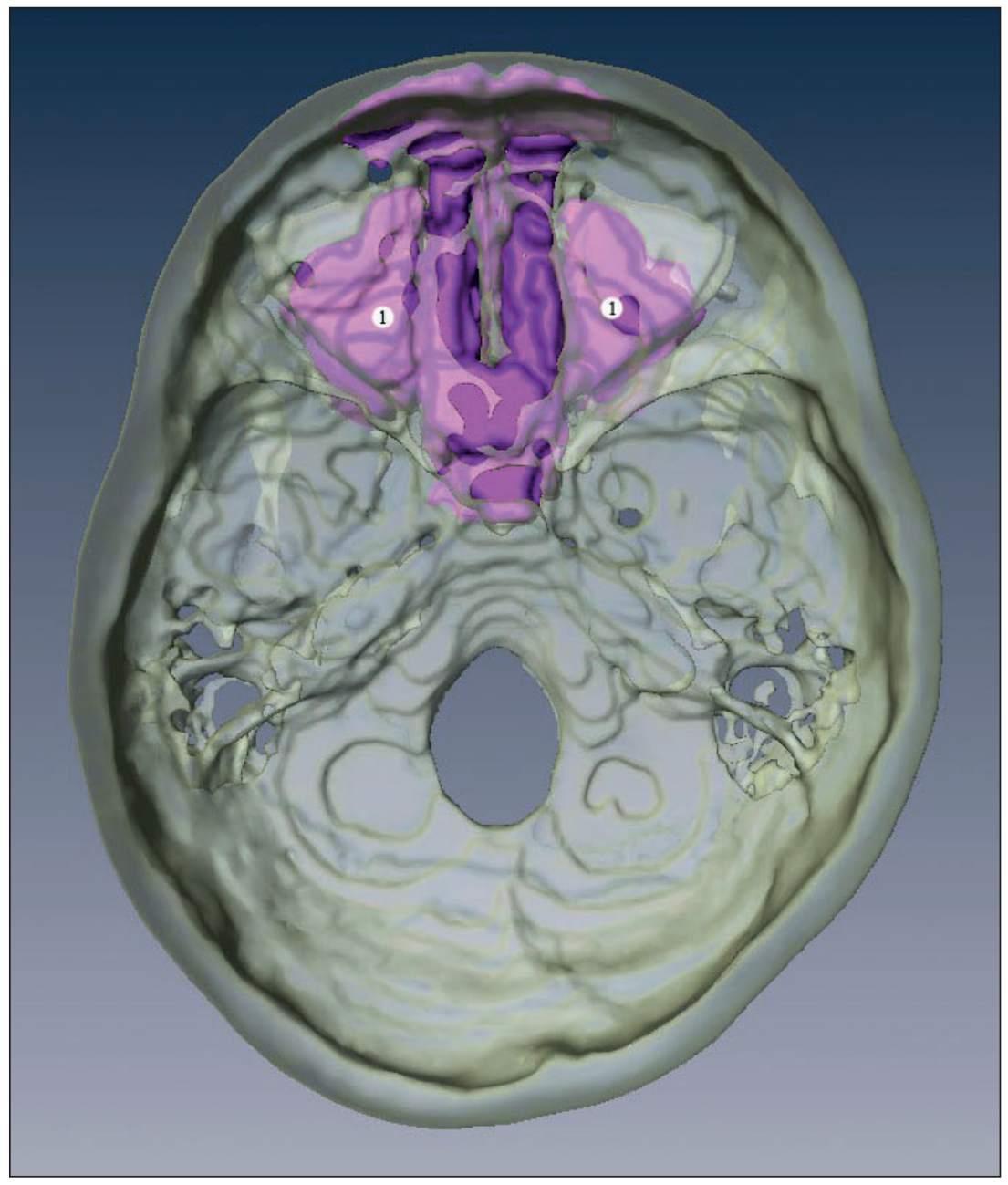

Fig. 1.3-D model of the infant's skull and paranasal sinuses. 1 - maxillary sinuses.

development and structure of maxillary sinus to improve the diagnosis and treatment of this disease $[8,9,10,11,12]$.

\section{THE AIM}

To study the peculiarities of the structure and to see the development of maxillary sinuses in infants, during the early and first childhood periods of human ontogenesis.

\section{MATERIALS AND METHODS}

The study of the peculiarities of the development and formation of the MS walls of the is carried out on 50 specimens of the upper jaws, turtles and sections of the corpses of the infants, children in early and first periods of human ontogenesis by histological examination, preparation, CT scan, radiography and morphometry.

\section{RESULTS}

In infants (10 days - 1 year), MS in all investigated specimens are located more lateral to the base of the lower nasal concha. In this period its lower wall begins to develop. It has a shape of a narrow strip, which invaginates into the alveolar process, forming alveolar special space. Its vertical size is $3.0-4.0 \mathrm{~mm}$, transverse $-2.0-2.6 \mathrm{~mm}$, anterior-posterior $-10.0-12.5 \mathrm{~mm}$. From the lower and middle nasal passages, the sinus is located at a distance of $3.5-3.6 \mathrm{~mm}$, from the lower wall of the ocular fossa $-2.0-2.1 \mathrm{~mm}$. The lower wall of the sinus is narrow. Its medium wall corresponds to the average nasal passage and is represented by a duplication of the respiratory mucous membrane (mucosa). The anterior wall of the sinus borders with the posterior margin of the nasopharyngeal canal.

The growth of the sinus is due to the protrusion of the external wall in the direction of the zygomatic bone. The bottom of the sinuses and the upper (очноямкова) wall are developed from the external wall. The lateral part of the sinus gives rise to two walls - the anterior and posterior (Fig. 1).

The walls of the MS are covered with mucosa. It is lined by pseudostratified columnar ciliated epithelium, which is located on the basement membrane.

The thickness of the respiratory mucous membrane is $0.5-0.65 \mathrm{~mm}$. Because of pneumatization of MS there is formation of its mucous glands. Already are found com- 


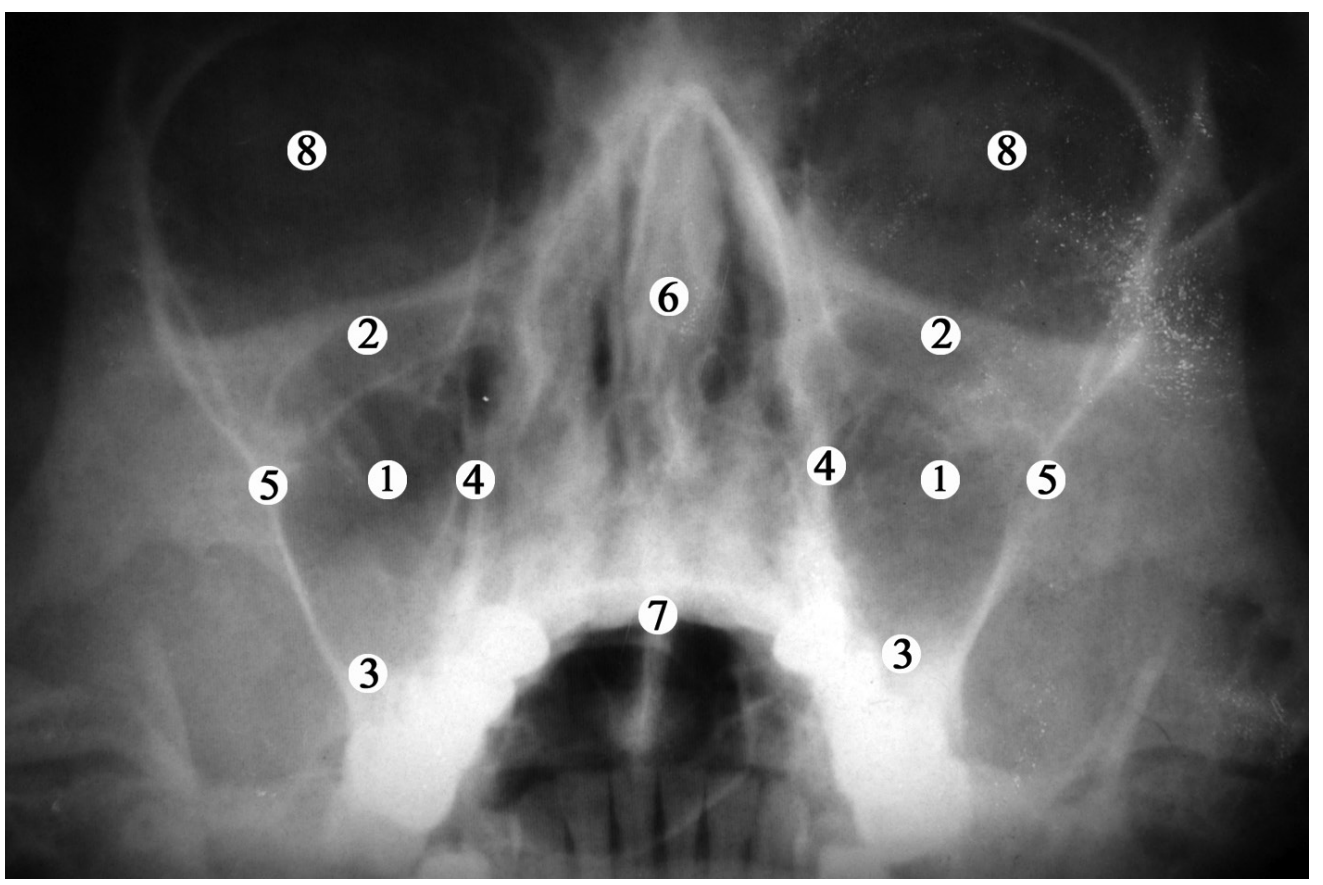

Fig. 2. X-rays of 7-th old girl.

1 - maxillary sinuses;

2 - upper walls of the sinuses;

3 - lower walls of the sinuses;

4 - median walls of the sinuses;

5 - posterior walls of the sinuses;

6 - nasal septum;

7 - hard palate;

8 - orbits.

pound tubular-alveolar glands. The glands of the subepithelial layer of the mucosa of the maxillary sinus are located by separate rows. The greatest concentration of glands is observed on the medial wall of the sinus, especially in the sinus openings, which border with the mucosa glands of the of the middle nasal meatus.

$\mathrm{X}$-ray of infants' MS in the anterior projection is pearshaped. Clearly the following walls of the sinuses are visible: the upper, the lower, one, which is arched-shaped, the front and the median, which in lower part are crossed as arch to each other.

Investigation of biological specimens of the maxillofacial area in the early childhood period (1-3 years) showed that MS are located more laterally to the base of the lower nasal concha. Its vertical dimension is 7.5-8.0 mm, transverse 5.7-6.0 mm, anterior-posterior - 13.9-14.5 mm. From the lower and middle nasal meatuses, it is located at a distance of 4.5-4.6 mm, from the lower wall of the orbit - 2.4-2.5 $\mathrm{mm}$. The lower wall of the sinus was wider. The median wall corresponded to the average nasal meatus and is presented as in the previous stages of development, by a duplication of the respiratory mucous membrane. The walls of the MS are covered with mucosa. The respiratory mucous membrane is lined by pseudostratified columnar ciliated epithelium, which is located on the basement membrane. The thickness of the mucous membrane reaches 1.0-1.65 mm. Compound tubular-alveolar glands are present in mucosa. The glands of the subepithelial layer of the MS mucous membrane are arranged in separate rows. The most concentration of glands was detected on the medial sinus wall, which were bordered with the glands of the mucous membrane of the middle nasal meatus.

$\mathrm{X}$-ray examination showed that MS in the front projection had an oval shape in early childhood. There are the following sinus walls: upper, lower (arched shape), anterior and median.
The study of biological specimens of the facial area in the period of the first childhood (4-7 years) has been established that the configuration of MS is changing. All its walls are determined, but the upper wall is rather short, and the front is narrow. The median wall was the most prominent.

Growth with expansion of sinuses in depth, to the caudal appendage, to the front and to the back is observed. At the end of the period of early childhood, the sinuses in the back touch to the alveolar of the sixth and seventh teeth, in the front it drops below the attachment place of the nasal concha, the bottom reaches alveolar would of canine and touches the second premolar alveolar.

On X-rays in the anterior projection, a slight extension of the MS is seen laterally. In the side of the anterior wall of the sinus, an oval foramen is determined, in the upper wall is visible infraorbital canal with a diameter of $1.5 \mathrm{~mm}$. There are also well developed rudiments of the teeth, which occupy almost the whole body of the upper jaw. The light places in the area of tooth alveolar are very small and are difficult to be determined (Fig. 2).

At the age of 4-7 years on the X-rays in the anterior projection clearly is visible expansion of the sinuses in the direction of the zygomatic bone. The bottom is good visible as a gap of 1-1.5 $\mathrm{mm}$ in width. In all X-rays there is a noticeable increasing of the teeth height and is well seen that they are projected to all region of the MS. The changes in the position of the anterior sinus wall are also manifested: in the middle third it has parallel orientation to the posterior one. In this case, the upper wall along the length is equal to the front.

In one case, the left MS was almost twice as low as the right one. MS was located at the level of the middle nasal meatus, the infraorbital canal was projected on the level of the upper and anterior sinus walls.

In children from 4 to 7 years old, a general increasing of the MS size is noted, it is visible the next growth in the 
height of the rudiments of the teeth and filling by them the space of MS. In the anterior projection of the X-rays it is possible to see only weakly expressed upper sections of the outer walls of the MS. All other sinuses' boundaries, including its bottom, are not visible due to the overlapping of shadows of teeth on them.

Thus, the period of teething of the baby-teeth and their functions from 4 to 7 years on the X-rays are characterized by the continuous growth of the walls of the MS and the increasing of their volume. The close anatomical connection of the MS with the rudiments of the baby-teeth and then the permanent teeth, is determined.

The rudiments of the teeth with the crowns touch to each other, taking the step-like arrangement, filling the lower half of the lumen of the MS at the end of this age period.

Thus, the growth of MS occurs in the lateral direction - towards the zygomaticus process. MS are increasingly removed from the alveoli of the tooth rudiments, pneumatization of its upper parts increases.

In the anterior projection, the sinuses are defined between the upper part of the medial region of the orbit and the lateral wall of the nasal cavity, whereas the комірки of the dental rudiments are located much lower, they have spherical shape, in their bottom the rudiments of the teeth with acute triangular projections in the upper sections are differentiate.

\section{DISCUSSION}

According to the research by Rossie J.O. [13] the upper jaw appears least «ready», its development and growth occurs after birth, sinuses develop as a result of resorption of spongy bone and the growth in to the diverticula of the nasal mucosa. According to our research the MS is located laterally in the base of the lower nasal concha in the period of 10 days -1 year. In this period begins to form its lower wall, which as a narrow strip invaginates into the alveolar process. The growth of the sinus is due to the protrusion of the external wall in the direction of the zygomatic bone. The walls of the MS are covered with respiratory mucous membranes. The mucosa is lined with pseudostratified columnar ciliated epithelium, which is located on the basement membrane.

The questions of individual and typical variability of the nasal cavity and sinuses sizes are still poorly studied. In particular, neither anthropologists nor clinicians have established correlations of parameters of the nasal cavity with the size and volume of the sinuses $[14,15,16,17]$. Were analyzed the 60 CT scan (age of patients 1-60 and more years) in order to study age-related changes in MS, based on an analysis of the three-dimensional models. After birth, there are two periods of rapid growth of the MS: the first 3 years of life and the period from 7 to 12 years, and according to our research in children aged 4 to 7 years, a general increase in the size of the MS and the rapid growth of all their walls are noted. The period of teething of baby-teeth and their functions from 4 to 7 years on radiographs are characterized by the continuous growth of the walls of the MS and the increasing in their volume $[18,19,20]$. The close anatomical connection of the MS with the rudiments of the baby-teeth and then the permanent teeth, is determined.

The inflammation of MS occurs more often in childhood and old age, but among children boys are more often affected. According to our investigations, up to $50 \%$ of children with maxillary sinusitis continue to be ill in adulthood, in the mechanisms of development of the majority of pathological processes, the additional individual features of the organism, which developed during embryogenesis, become important.

\section{CONCLUSIONS}

Thus, in infants and during the early and first childhood periods of human ontogenesis in the MS there are changes in both quantitative and qualitative nature.

In infants (10 days - 1 year) growth of the sinuses is due to the protrusion of the external wall in the direction of the zygomaticus bone, radiographically in the forward projection MS are pear-shaped.

In children of early childhood (1-3 years), the following walls in the MS are clearly distinguishable: the upper, lower, which is arched, the anterior and medial, which are arched below each other, radiographically in the forward projection of the MS they are oval shaped.

In the period of the first childhood (4-7 years) all the walls of the MS are determined, but the upper wall is rather short, and the anterior is narrow. Also the general increasing of the MS size is note, the next steps in growth of the rudiments of the teeth include the filling of their considerable space of sinuses. The X-rays indicated that the most prominent was the medial wall, and MS was oval shaped.

Therefore, one should thoroughly study the peculiarities of the development and structure of the walls of the maxillary sinuses in order to prevent the development of complications and to achieve the treatment of inflammatory diseases of the MS in an optimal term.

\section{REFERENCES}

1. Sato K. Odontogenic maxillary sinusitis caused by a fractured tooth. Nippon Jibiinkoka Gakkai Kaiho. 2008;111(12):739-745.

2. Voitovych OV, Kamyshnyı̆ OM. Peculiarities of microbiological and immunological indices of human nose mucosa in conditions of manmade pollution. Mikrobiol. 2014;76(2):47-53.

3. Doty R, Mishra A. Olfaction and its alteration by nasal obstruction, rhinitis, and rhinosinusitis. Laryngoscope. 2012;111(3):409.

4. Durr DG, Desrosiers MY, Dassa C. Impact of rhinosinusitis in health care delivery: Quebec experience. J. Otolaryngol. 2011;30(2):93.

5. Cecil K, Tindall L, Haydon R. The relationship between dysphonia and sinusitis: a pilot study. J. Voice. 2009;15(2):270.

6. Drettner B, Aust R. Pathophysiology of the paranasal sinuses. Acta Otolaryngol. 2010;53(3):477-485.

7. Kuzniak NB, Dmytrenko RR, Fedoniuk LY et al. Development of the inner nasal cavity in animals in phylo- and ontogenesis: functional anatomic significance in the development period. Wiad Lek. 2019;72(3):432-435. 
8. Bomeli SR, Branstetter BF, Ferguson BJ. Frequency of a dental source for acute maxillary sinusitis. Laryngoscope. 2009;119(3):580-584.

9. Akhaddar A, Elasri F, Elouennass M. et al. Orbital abscess associated with sinusitis from odontogenic origin. Intern. Med. 2010; 49(5):523-524.

10. Wysluch A, Maurer P, Ast J. et al. Orbital complications due to an acute odontogenic focus in a child. A case report. Oral Surg. Oral Med. Oral Pathol. Oral Radiol. Endod. 2009;107(1):39-42.

11. Warwar RE, Rogers DL. Exophthalmos and orbital floor thickening related to maxillary sinusitis. Ophthal-Plast-Reconstr-Surg. 2010;19(2):158.

12. Bojchuk TM, Yermolenko SB, Fedonyuk LYa et al. The magnitude of linear dichroism of biological tissues as a result of cancer changes. Proc. SPIE. Materials of the 10th International Conference Correlation Optics. 2011;8338:K1-K7.

13. Rossi JB. Ontogeny and homology of the paranasal sinuses in Platyrrhini (Mammalia). J. Morph. 2006;267:1-40.

14. Rysz M, Bakoń L. Maxillary sinus anatomy variation and nasal cavity width: structural computed tomography imaging. Folia Morphol. 2009;68(4):260-264.

15. Churchill SE, Shackelford LL, Georgi JN et al. Morphological variation and airflow dynamics in the human nose. Am J Hum Biol. 2004;16(6):625-638.

16. Liu Y, Johnson MR, Matida E, et al. Creation of a standardized geometry of the human nasal cavity. J Appl Physiol. 2009;106:784-795.

17. Boiko V, Boychuk A, Nikitina I. et al. Basic clinical and pathogenetic aspects of developing the complications during multiple pregnancies. Wiad Lek. 2019;72(1):52-55.

18. Gambaruto AM, Taylor DJ, Doorly DJ. Decomposition and description of the nasal cavity form. Ann Biomed Eng. 2012;40(5):1142-59.

19. Lee CF, Abdullah MZ, Ahmad KA et al. Analytical Comparisons of Standardized Nasal Cavity. J Med Imaging Health Inf. 2014;4(1):14-20.

20. Boiko VI, Nikitina IN, Babar TV et al. The problem of miscarriage in multiple pregnancy. Wiad Lek. 2018;71(1):1195-1199.

The work is a fragment of inter-department al scientific research work of the Department of Human Anatomy named after M.G. Turkevichand The Department of Anatomy, Topographic Anatomy and Operative Surgery of the Higher State Education al Institution of Ukraine "Bukovinian State
Medical University" "Patterns of perinatal anatomy and embryo-typography. Determination of gender-age characteristics of the structure and topographic-anatomical interrelation in human ontogenesis" (state registration number 0110U003078).

\section{ORCID and contributionship:}

Nataliya B. Kuzniak - 0000-0002-4020-7597 A,B,D

Larisa Ya. Fedoniuk - 0000-0003-4910-6888 A,E,F

Antonina M. Pryshlyak - 0000-0003-4430-3735 E

Olena I. Skyba - 0000-0002-3826-8315 ${ }^{\mathrm{C}}$

Oksana M. Yarema - 0000-0002-6839-2253 ${ }^{\mathrm{C}}$

Alina I. Dovgalyuk - 0000-0003-3976-0245 ${ }^{\mathrm{E}}$

Nataliya P. Penteleichuk - 0000-0002-1850-9142 ${ }^{B, D}$

Vladyslav A. Smiianov - 0000-0001-8164-9706 ${ }^{F}$

\section{Conflicts of interest:}

Authors declare no conflict of interest.

\section{CORRESPONDING AUTHOR}

\section{Larisa Ya. Fedoniuk}

Head of the Medical Biology Department of the

I. Horbachevsky National Medical University

Maidan Voli, 1, 46000, Ternopil, Ukraine

tel: +380673999143

e-mail: fedonyuk22larisa@gmail.com

Received: 24.09 .2019

Accepted:04.01.2020

A - Work concept and design, B - Data collection and analysis, C - Responsibility for statistical analysis, D -Writing the article, E-Critical review, F- Final approval of the article 